\title{
Computerized Perinatal Database Management : Successes and Suggestions
}

\author{
Cynthia Ferre, Arden Handler and Deborah Rosenberg \\ Program in Community Health Sciences, School of Public Health, \\ University of Illinois, Chicago, Illinois, U.S.A.
}

Computers are now available worldwide for a multitude of functions. Over the past two decades, computers have become more powerful and affordable, and software has become more varied and "user friendly". In perinatal medicine, the use of computers has grown sharply since the early 1970 's. ${ }^{1}$ Medical record data management, fetal and neonatal monitoring, and neonatal screening surveillance are the most common applications of computers in perinatal medicine. This paper will discuss the use of computers for perinatal database management and offer suggestions for the development, implementation, and maintenance of such systems.

\section{Basis for Computer use}

The reasons for using computers in perinatal medicine are varied. The information generated by sophisticated medical services has grown exponentially in the last two decades as medical records have expanded to include risk assessments, detailed laboratory reports, and monitoring data. $^{2}$

Computers can collate diverse

Reprint requests : Dr. Cynthia Ferre, School of Public Health M/C 922, University of Illinois, Box 6998, Chicago, Illinois 60680, U.S.A. information which, due to specialization and regionalization of perinatal care, is often generated from multiple sources, ${ }^{3,4}$ Computerization can provide immediate access to medical records and integration of patient data for health care providers, eliminating delays in diagnosis and treatment resulting from chart assembly and review. ${ }^{3.5}$ Computerization confronts health care providers with the need for more complete charting and precise definitions of disease states and treatment procedures, which improves overall patient care and serves as a form of physician risk management. ${ }^{6}$ By linking data from gynecology to obstetrics to pediatrics through the creation of perinatal databases, computers enhance information flow and thus improve continuity of care, risk evaluation, quality of care assessment, and statistical analyses. $^{1-4,7-8}$

Perinatal databases may be general or specific. General databases coordinate and retain all medical record data for current or future use. Specific databases are created for special purposes, such as fetal monitoring or neonatal screening, and may be integrated with generalized databases.

\section{Medical Record Data Management}

Medical record database management is 
perhaps the most frequent use of computerization in perinatal medicine. Perinatal databases have been successfully used since 1973, and their use has especially increased since the development of microcomputers. ${ }^{9}$ The history of computer use for perinatal database management demonstrates that computers can directly improve clinical functioning and medical care.

Perinatal database management systems, whether retrospective or "on-line", integrate antepartum risk assessments, laboratory results, and ultrasound evaluations with labor, delivery and neonatal data. Printouts of records are easily available. Prospective systems can be programmed to automatically print discharge summaries, birth and death certificates, referral letters, and patient audits. ${ }^{9}$ Computer systems can also perform administrative tasks, such as managing clinic and teaching schedules, patient scheduling, and maintaining medical reference filling systems. ${ }^{1.2}$ Perinatal record databases can be prospective of retrospective. Computerized prospective databases are created by having current medical records "on-line" and available for use during current care. These records are stored on the computer for further use and analysis when care is completed. Retrospective databases are created by abstracting paper medical records after care has ended. Combinations of methods can also occur. New and current patients can be entered prospectively into the database while records from former patients are abstracted and entered retrospectively.,

There are many microcomputer hardware and software options available for the design of perinatal databases. Systems based on "floppy" (removable) disks are possible, and can be upgraded to hard disk (fixed) systems as needs and patient populations grow. ${ }^{10}$ The costs of hard disk systems have decreased, making them very affordable. A variety of low cost, commercial, easily used database management software programs, such as rBase, dBase III or IV, Database, and Informix are available. Custom-designed software may also be obtained for special purposes. Management of clinics with 1000 births per year is entirely possible using dBase III software on an IBM-PC with 512 kilobyte random access memory (RAM) and a 10 megabyte (MB) hard disk."

Computing resources may be shared among health facilities. Microcomputers can be linked together, to mini or mainframe computers via telephone lines to create local area networks (LAN) and aggregate community databases for statistical analyses. ${ }^{6,12}$ Data storage, maintenance, and reporting can be divided among several small computers while allowing data sharing among all members. ${ }^{12}$ This type of system reduces overall costs by distributing hardware, software and personnel costs among participants and provides the necessary data for reporting community statistics and research.

Perinatal databases exist at all levels of health care : office-based; clinic-based; hospital-based; regional; state; or national; and may even be shared among groups of nations. ${ }^{2,68,12-14}$ The Latin American Center for Perinatology and Human Development uses a one-page standardized Perinatal Clinical Record (SPCR) as a planning tool for perinatal health coverage in 13 countries, collecting data on maternal history and risk factors, labor and delivery and neonates. Computer facilities are provided locally so data is entered by those collect- 
ing the information, facilitating local correction and processing for immediate use. Use of the same form in all participating institutions anticipates merging data for epidemiologic studies. ${ }^{13-14}$

\section{Fetal and Neonatal Monitoring Databases}

The ability of computers to capture, store and process complex signals from electronic devices makes them valuable in perinatal monitoring. ${ }^{2-3,15}$ Examples of computerized monitoring include ultrasound evaluations, detection of uterine contractions from background noises, CAT scanning, monitoring of fetal heart rate and neonatal respiration and home monitoring of maternal blood glucose levels. ${ }^{16-18}$ Data generated from monitors is stored electronically to produce patient-specific data files. Computerized monitoring systems can coordinate the simultaneous data produced on several parameters, such as airway pressure and flow and calculate physiologically significant variables and trends, such as respiration rate. ${ }^{2.15}$ This facilitates clinical decision making and treatment because important clinical factors and relationships can be detected earlier. ${ }^{2}$ Once these data are collected and stored, computers can reconstruct events for further evaluation. ${ }^{2,18}$

Computer analysis reduces time involvement, observer fatigue and calculation errors because defined criteria can be applied repeatedly and persistently. ${ }^{2}$ Computerized monitoring can thus lead to fewer management errors during the care of high risk infants. The same system can also generate output for medical records, patient billing and quality of care assessment. ${ }^{19}$

Neonatal Screening Surveillance

Computerized databases in the U.S. facilitate newborn screening for phenylketonuria (PKU), galactosemia, hypothyroidism, biotinidase deficiency and other genetic diseases by coordinating the activities of the practitioner, screening laboratory and follow-up team. ${ }^{20}$ Computerized system are advantageous because such testing generates large quantities of data, requiring both repetitive analytical calculations and linkage of individual results, reports and follow-up which would be difficult to manage with paper-based systems. ${ }^{21}$ ${ }^{22}$ State or regional computerized systems for neonatal screening surveillance assure that all newborns are tested, tracked and provided follow-up services if screening results are positive. Computers are also used for report generation, automatic mailings, revenue collection and quality control evaluations. ${ }^{20-22}$

Computerized data are also used to improve testing procedures and to understand physiological processes. Laboratories with unusual error rates are identifiable by database analysis. An association between low birth weight and high false positive rates for hypothyroidism and PKU was detected in analysis of the California screening database. ${ }^{22}$ The database provided actual $\mathrm{T} 4$, TSH, and phenylalanine levels for the establishment of new cutpoints for presumptive positives at low birth weights.

\section{Developing a Computerized Perinatal Database}

Most suggestions offered by experienced perinatal database creators for system development concern guaranteeing the completeness, accuracy and utility of the data collected. To achieve this, a comprehensive plan concerning how data collection, entry, storage, use and evaluation will occur must be conceived in the preliminary 
stages of development. Whether the system will be prospective or retrospective will help guide design and planning, as will hardware costs.

While each database is designed to meet unique needs, general suggestions for computerized perinatal system development are outlined below.

1. The goals of the system should be well-defined. Projects should start small and grow only with increasing experience and acceptance. Completely computerized systems can be created by linking several smaller programs together over time. ${ }^{2}$

2. User acceptance is crucial. ${ }^{24}$ Explicit collaboration between'physicians and other personnel using the database should be formally obtained. Health care providers need to view the computerized database as an ordinary aspect of patient care. To achieve this, providers must be included in all planning stages, such as choice of hardware and software, form designs and program contents.

Using the computer system to provide a quick service, such as computer-generated discharge summaries or referral letters, can build acceptance and familiarity while the capabilities of the system to accomplish more difficult tasks. are developed.

3. Ease of use is also critical. On-line operations should be simple. ${ }^{2,4}$ Menudriven screens are helpful for easing program use. Such "user-friendly" systems permit accessibility to the database with a minimum amount of training for physicians and other health care personnel.

Enough terminals should be available to facilitate data entry and retrieval. The computer should have adequate processing speed for data retrieval to occur rapidly.

4. Adequate electrical power protection and data backup are crucial. ${ }^{210}$ These capabilities ensure computer functioning and avoid loss of information when problems do occur (and they will). Protected power supplies are obtained by the use of surge protectors, power line conditioners, and even power generators. Data backup includes periodic printing of paper copy, copying to magnetic tapes and duplicate storage on backup computer systems.

5. An assessment should be conducted to establish which data are to be collected and what reports or audits will be generated. Variables should be identified and definitions standardized. The flow of data through the system should be conceptualized. Flow charts or diagrams may help in this process. Methods should be developed to ensure reports will be produced promptly.

6. Before the database is considered "up"; a pilot stage or "trial run" should occur. This enables identification of previously unforeseen problems and allows major system revisions to occur before the database is fully operational.

A plan for ongoing, continuing revisions and updating of database procedures should occur beyond the pilot stage. Regular meetings of all personnel (or representatives) should be scheduled to discuss the database operation and problems encountered.

7. Adequate programming and technical expertise.are required. A program for training and monitoring personnel (i.e., medical record abstracters and data entry staff) should exist.

8. Assessment of data quality should be incorporated into the design of the system. This involves checks on data and reports, audits and feedback from database personnel. Data quality in perinatal databases is considered in more depth in the 
article by Rosenberg et $\mathrm{al}^{23}$ in this issue.

9. Perinatal database developers should be candid about problems encountered. ${ }^{2}$ Networking with other databases can be beneficial. Since the time involved in successfully introducing a perinatal database system and training personnel can be considerable, long-range plans must be kept in perspective. ${ }^{24}$

\section{Discussion}

There have been numerous special applications of computers in perinatal medicine, such as automated therapeutic intervention, computer-based education, patient interviewing, and differential diagnosis using artificial intelligence or "expert systems". Computerized treatment intervention has improved perinatal survival and care in neonatal intensive care (NICU) infants. Computers can precisely calculated and administer ventilator support, drug dosages and nutritional requirements as well as regulate incubator temperature and wean infants from incubators to nursery ambient climates. ${ }^{3,19,25}$

The uses of computers in interviewing and diagnosis has not yet received widespread acceptability or success. Harris argues this unacceptability, and says that the use of computers add a dehumanizing element to medical care and remove an aspect of medicine which the clinicians are skillful at and enjoy, namely problem solving and diagnosis. $^{2}$

More enthusiasm exists for the use of computers in the management of data collected during the course of medical care, whether in medical record databases, fetal and neonatal monitoring, therapeutic interventions, or neonatal screening surveillance. In these applications, computers supplement the skills of health care provid- ers with improved data integration, accessibility and precision during medical diagnosis and treatment. Skillful use of perinatal database management and building up on clinical expertise, will ultimately lead to better patient care in a cost-effective manner.

\section{RERERENCES}

1. Sokol RJ, Zador IE. Perinatal computing in 1987 : an overview. J Perinatal Med 1987; 15 (Suppl 1) : 5-6.

2. Harris TR. The use of computers in perinatal medicine. In : Harris TR, Bhar JP, ed. The Use of Computers in Perinatal Medicine. New York : Praeger Pub, 1982 : 3-22.

3. Bahr JP, Grausz JP. Real-time analysis of patient data. In : Harris TR, Bahr JP, ed. The Use of Computers in Perinatal Medicine. New York : Praeger Pub, 1982 : 311-326.

4. Frayer WW. Patient data management in neonatal intensive care. Clin Perinat 1980; $7: 145-154$.

5. Rock JA, Early SA, Zacur HA, Wallach EE. A computer-based system for patient care and research management in reproductive endocrinology. Fertil and Steril $1986 ; 45: 216-220$.

6. Raju TNK, Vidyasagar D, Wineagar A. Early development of regionalized perinatal database : the Chicago experience. In : Harris TR, Bahr JP, ed. The Use of Computers in Perinatal Medicine. New York Praeger Pub, 1982; 297-308.

7. Nakahara $\mathrm{H}$, Koyanagi $\mathrm{T}$, Hirose $\mathrm{K}$, Nakano H. Assessment of local area network based on the microcomputer system for data processing of perinatal medical information. J Perinatal Med 1988; 16 : 315-318.

8. Jennett RJ, Gall D, Waterkotte GW, Warford HS. A computerized perinatal data system for a region. Am J Obstet and Gynecol 1978; 131 : 157-161. 
9. Maresh M, Beard RW, Combe D et al. Selection of an obstetric data base for a microcomputer and its use for on-line production of birth notification forms, discharge summaries and perinatal audit. Br J Obstet and Gynecol 1983; 90 : 227-231.

10. Houlton M, Austin J, Jenkins D et al. A microcomputer system in the delivery suite. Br J Obstet and Gynecol 1984; 91 : 555-559.

11. Pluta $M$. Perinatal data base including perinatal basic documentation and ICDclassification. J Perinatal Med 1987; 15 (Suppl 1) : 20.

12. Andersen HF, Damus $K$, Parnian $M$, Merkatz IR. A country-wide distributed perinatal database. $J$ Perinatal Med 1987; 15 (Suppl 1) : 8.

13. Schwarcz, R, Diaz AG, Pascina $R$ et al The perinatal information system $I$ : the simplified perinatal clinical record (SPCR). J Perinatal Med 1987; 15 (Suppl 1) : 9.

14. Simini F, Diaz AG, Lopez R, Schwarcz R. The perinatal information system II : an instrument for epidemiologic control. $J$ Perinatal Med 1987; 15 (Suppl 1) : 131.

15. Lindstrom $D$, Cotton $R$. Use of computers in the newborn intensive care unit. Clin in Perinatology 1983; $10: 195-203$.

16. Mendez-Bauer C, Tran B, Schumann S, Freese U. A new development in fetal tracing storage and retrieval. $J$ Perinatal Med 1987; 15 (Suppl 1) : 106.

17. Dalton $\mathrm{KJ}$, Alban-Davies $\mathrm{H}$, Nicholls $\mathrm{J}$ et al. Computerized home telemetry of ma- ternal blood glucose levels in diabetic pregnancy. J Perinatal Med 1987; 15 (Suppl 1) : 93.

18. Ott WJ. The design and implementation of a computer-based ultrasound data system. J Ultrasound Med 1986; 5: 25-32.

19. Perlstein PH, Edwards NK, Atherton HD, Hermansen MC. Computer-assisted environmental control for newborns. In : Harris TR, Bahr JP, ed. The Use of Computers in Perinatal Medicine. New York : Praeger Pub, 1982: 363-373.

20. Udell BD, Crouch J, Medel R. Interactive parenteral nutrition program for newborns. J Perinatal Med 1987; 15 (Suppl 1) : 28.

21. Meaney FJ. Computerized tracking for newborn screening and follow-up: a review. J Medical Systems 1988; 12 : 69-75.

22. Kling S, Nash C, Jones D. Newborn screening in the 80's-the automation of follow-up. J Medical Systems 1988; 12 : 89-96.

23. Mordaunt VL, Cunningham GC, $\operatorname{Kan} K$. Computer assisted management of a regionalized newborn screening program. $J$ Medical Systems 1988; 12 : 77-88.

24. Rosenberg D, Rohodes C, Ferre C, Handler A. Insuring quality in a computerized database system: an example from a perinatal network. Indian J Pediatr 1989; 56 :

25. Macagno F, Furlan R, Cattarossi L, Bortolin M. Quality care evaluation in very low birth weight infants VLBW: Assessment using a personal computer on $\mathbf{3 3 0}$ VLBW sample. $J$ Perinatal Med 1987; 15 (Suppl 1) : 60 . 